\title{
Effects of Piano Music of Different Tempos on Heart Rate and Autonomic Nervous System During the Recovery Period After High-intensity Exercise
}

\author{
Biyun Xue \\ Henan Normal University \\ Jiameng Wang ( $\sim$ hy0215037@hainmc.edu.cn ) \\ Hainan Medical University
}

\section{Research Article}

Keywords: High-intensity exercise, Piano music, Heart rate variability, Sympathetic nerve, Parasympathetic nerve

Posted Date: January 18th, 2022

DOI: https://doi.org/10.21203/rs.3.rs-1227474/v1

License: (c) (1) This work is licensed under a Creative Commons Attribution 4.0 International License. Read Full License 


\section{Abstract}

Purpose: The present study attempted to explore the effects of different tempos of piano music on heart rate and autonomous nervous system during the recovery period after high-intensity exercise. In addition, the study analyzed the influence of different tempos on the recovery period of athletes to devise methods for accelerating fatigue recovery through piano music.

Method: A total of 57 college students majoring in physical education were selected as experimental subjects and were divided into three groups, namely Lento group $(n=20)$, Moderato group $(n=20)$, and Allegretto group $(n=20$; only 17 students completed the experiment).

Results: Under the same high-intensity exercise regimen, the three groups did not differ significantly in the body composition, high-intensity exercise ability, and time-domain variation indices, namely heart rate (HR), Heart rate variability index parameters( $P$ $>$.05). The time-domain variation analysis in the recovery period revealed significant differences in HR frequency domain indices among the groups exposed to different rhythms $(P<.05)$.

Conclusion: Moderate-tempo piano music was the most effective in facilitating HR and autonomic nervous system recovery during the recovery period.

\section{Background}

Fatigue is a common physiological phenomenon observed in athletes. The mechanisms of fatigue in sports have been described through various theories, such as energy exhaustion theory, protective inhibition theory, blockage theory, internal environment stability disorder theory, and free radical theory ${ }^{1}$. According to the Pavlov school, exercise conducts nerve impulses to the cerebral cortex, which leads to long-term excitation and increased energy consumption. Additionally, the body inhibits the cerebral cortex for self-protection, which leads to exercise-induced fatigue ${ }^{2}$. The application of external intervention is conducive to fatigue recovery, though exercise-induced fatigue can restore normal physical functions after a period of rest and adjustment ${ }^{3}$.

Heart rate (HR) variability reflects the variation in the heartbeat cycle and can be used to analyze the successive heart cycle time difference. HR is regulated by the autonomic nervous system (ANS). Consequently, HR variability is a noninvasive index that quantitatively reflects the dynamic balance of cardiovascular regulation by the ANS ${ }^{4}$. The ANS, a part of the peripheral efferent nervous system, can regulate the activity of visceral and vascular smooth muscles, myocardium, and glands. The autonomic nerve can be divided into sympathetic and parasympathetic nerves, which innervate the common internal organs. However, the signals from these nerves are antagonistic. For most organs such as the heart, the sympathetic nerves excite the organs, whereas the parasympathetic nerves excite and suppress the organs ${ }^{5}$.

According to studies, music and exercise affect the ANS activity ${ }^{5}$. Sympathetic activity and stimulation increase during sports, which increases the HR, stroke volume output, and systemic vasoconstriction ${ }^{5}$, whereas exercise decreases the parasympathetic activity, which increases the HR. However, the HR decreases rapidly after exercise ${ }^{6}$. Rapid HR recovery plays a crucial role in preventing cardiac overwork after exercise. The decrease in the HR after exercise is mediated by the increased parasympathetic activity and decreased sympathetic activity. Several studies have revealed that delayed activation of the parasympathetic nerve after exercise may increase the risk of sudden cardiac death. Therefore, parasympathetic reactivation after exercise is a crucial mechanism for protecting the heart of healthy individuals and patients ${ }^{7,8}$.

Music can effectively regulate the mood and ANS activity. Moreover, it serves as a potentially economical and safe interventional and treatment method ${ }^{9}$. Studies have revealed that sedative music exerts dual effects of high subjective relaxation and low tension in young adults. The relaxation effect of music can stimulate or strengthen the autonomic nerve balance and parasympathetic innervation, thereby stabilizing the ANS state ${ }^{10}$. In a study, the group listening to high-speed music exhibited stimulation in their parasympathetic activity more effectively than the control group exposed to a quiet environment ${ }^{11}$.

Thus, music can regulate the ANS activity after exercise. The body's acceptance of different music types might affect the parasympathetic nervous system differently. For example, studies have analyzed this phenomenon by asking participants to

Page 2/12 
listen to their favorite music. However, these studies did not consider the type and tempo of music. The music consists of various components, such as melody, tempo, and harmony. Among these components, the tempo is the primary factor that causes changes in the parasympathetic nervous system ${ }^{12,13}$. Although a few studies have already analyzed the effects of different music tempos on the ANS activity after exercise, the current study attempted to verify whether different music tempos have a related impact on the HR and HRV system during the recovery period after high-intensity exercise among athletes. The current study also investigates the most effective music tempo for the recovery of ANS and HR after exercise to develop methods for accelerating fatigue recovery.

\section{Methods}

\section{Participants}

The stratified cluster random sampling method was used to select 100 college students from a university in Hainan province as the research objects to performed on the power bike and monitor the indexes of autonomic nervous system. In this research,student selection was based on achievement test scores, 60 students with little difference in scores were selected as the research subjects.Finally, we used one-way ANOVA test and adjusted the grouping to ensure that there was no significant difference in baseline characteristics between the groups. Thus, A total of 60 college students majoring in physical education were selected as research subjects and were divided into three groups: Lento group $(n=20)$, Moderato group $(n=20)$, and Allegretto group $(n=17)$. Three students could not complete the experiment due to some reasons in Allegretto group. The experiment was performed after an inform consent form was signed by each participant to the study and confirming that none of the students had cardiovascular and other lethal diseases. The study was conducted in accordance with the Declaration of Helsinki and approved by the Bioethics Commission of the Hainan Medical university. The fundamental physical characteristics of each group are shown in Table 1.

Table 1

Basic physical characteristics of the participants

\begin{tabular}{|lllll|}
\hline Subjects & Age(yrs $)$ & Height(cm) & Weight(kg) & BMI $\left(\mathrm{kg} / \mathrm{m}^{2}\right)$ \\
& $M(S D)$ & $M(S D)$ & $M(S D)$ & $M(S D)$ \\
\hline lento $(n=20)$ & $22.20(1.77)$ & $172.35(8.27)$ & $72.38(15.92)$ & $23.97(3.62)$ \\
\hline Moderato $(n=20)$ & $23.80(2.93)$ & $172.95(6.27)$ & $69.11(11.23)$ & $22.90(2.78)$ \\
\hline Allegretto $(n=17)$ & $23.35(2.85)$ & $172.26(8.58)$ & $72.64(15.42)$ & $24.40(3.62)$ \\
\hline
\end{tabular}

\section{Research methods}

QThe body composition tester (model: inbody720, Manufacturing country: Korea) was used to analyze the body composition of the participants. In addition, the HR variability index of the participants was measured before exercise by using the heart rate variability measurement system UbpuseT (model: UbpuseT1; manufacturing country: South Korea). The heart rate variation of each group was recorded and compared when the group was quiet.

\The power bike(model: EXCALIBUR; manufacturing country: South Korea)was adopted; load was set to 0.070kp/kg, with warmup at $60 \mathrm{watt} / \mathrm{min}$ speed for $120 \mathrm{~s}$, and then pedaled at the maximum speed for $30 \mathrm{~s}$. Anaerobic power indicators such as mean power (MP), peak power (PP), and fatigue index (FI) were recorded.

QHeadphones (Sony WH-1000XM3, manufacturer: Japan) were put on immediately, and they listened to the piano repertoire of the corresponding speed according to the group. Information on the selected repertoire is presented in Table 3. 
Table 2

Repertoire information

\begin{tabular}{|llll|}
\hline Repertoire name & Approximate speed beat/minute & Speed mark & Length of time \\
\hline Chopin Etude, Op.25, No.7 & 52 & Lento & 5 minutes 20 seconds \\
\hline Schumann: Kinderszenen, Dreaming, Op.15 No.7 & 88 & Moderato & 3 minutes 10 seconds \\
\hline Mozart Sonata in C Major, Movement III, K.330 & $108-126$ & Allegretto & 5 minutes \\
\hline
\end{tabular}

We started to press the timer, and used UbpuseT1 to test the heart rate variability index at the same time. Besides, the heart rate variability index was measured 1 minute later. The subjects were asked to listen to the music, lying on the prepared bed for 5 minutes. The heart rate variability index was measured again, and the test was finished 6 minutes later; They kept the same Repertoire and lied on the bed for 10 minutes, then the heart rate variability index was measured again, and the test was finished 11 minutes later; The heart rate variability index was measured again at 15 minutes, and the test was completed after 16 minutes. Besides, the heart rate variability index was measured in a sitting position when measuring the heart rate variability index.Table 4 Analysis of the HR variability index.

Table 3

Heart rate variability index parameters

\begin{tabular}{|lll|}
\hline $\begin{array}{l}\text { Heart rate variability } \\
\text { index }\end{array}$ & Definition & Parameter meaning \\
\hline HR (times/min) & Average heart rate & Reflecting the average level of RR interval \\
\hline SDNN (ms) & $\begin{array}{l}\text { Standard deviation of all normal adjacent } \\
\text { RR intervals }\end{array}$ & $\begin{array}{l}\text { A relatively simple index to evaluate the activity of } \\
\text { autonomic nerves. }\end{array}$ \\
\hline LF (ms2) & Low-frequency power & Reflecting the activity of the sympathetic nerve. \\
\hline HF (ms) & High-frequency power & Reflecting the activity of the parasympathetic nerve. \\
\hline LFnorm (n.u.) & LF/(LF+HF)*100 & $\begin{array}{l}\text { Directly reflecting the changes of sympathetic } \\
\text { regulation. }\end{array}$ \\
\hline HFnorm (n.u.) & HF/(LF+HF)*100 & $\begin{array}{l}\text { Directly reflecting the changes of parasympathetic } \\
\text { nerve regulation. }\end{array}$ \\
\hline LF/HF & Low-frequency power / high-frequency & $\begin{array}{l}\text { The balance of sympathetic and parasympathetic } \\
\text { nerves }\end{array}$ \\
\hline
\end{tabular}

Note: The environment and time of this experiment were kept fixed, the laboratory temperature was maintained at $23^{\circ} \mathrm{C}$, the relative humidity was $65 \%$, and the experiments were performed daily between 14:00-15:00 of the day to minimize the influence of external factors on the results. The participants were prohibited from listening to other music, performing strenuous exercise, or eating and drinking caffeinated food $3 \mathrm{~h}$ before the experiment. Additionally, the participants were prohibited from drinking alcohol $48 \mathrm{~h}$ before the experiment. We controlled the test speed of the three people to be measured every day, and the volume was uniformly adjusted to 60 decibels. The repertoire was set on the single-cycle mode and played continually. The data were recorded and saved at the same time during the 0-16 min period.

\section{Data analysis}

SPSS2 version 22.0 was used for data processing, and the average value of the body characteristic parameters, including body composition, was calculated Wingate test and HRV index of each group of data was worth the standard deviation. Repeated oneway ANOVA was adopted to analyze differences in the body composition, Wingate index, and pre-exercise HRV indices among the three groups. The differences in HRV indices among the three repertoire groups during the recovery period were analyzed using repeated two-way ANOVA and test LSD. The hypothesis test standard for the determination of differences in parameters among the groups was set as $P<.05$. 
Results

\section{Analysis of differences in the body composition and Wingate Power Bicycle indices among the groups}

Table 4

Analysis of body composition differences among the groups

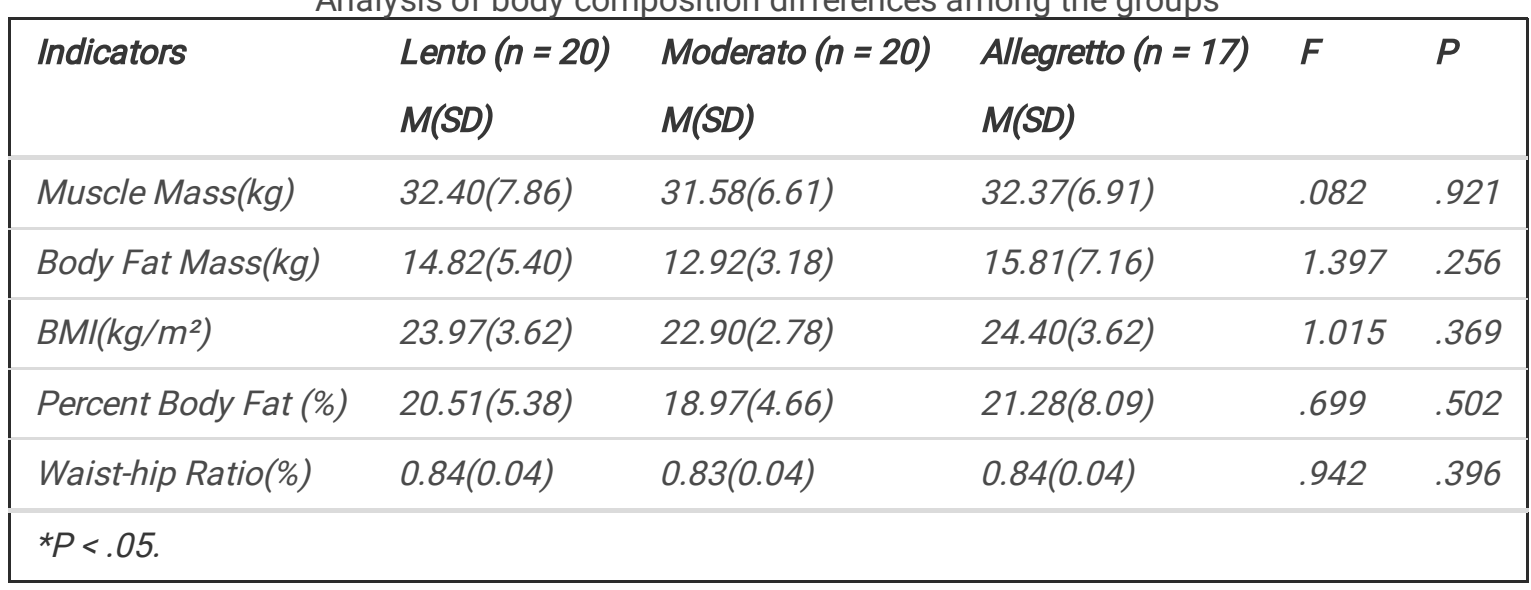

Table 4 presents the body composition analysis results. The three groups exhibited no significant difference in muscle mass, body fat mass, $\mathrm{BMI}$, percent body fat, and waist-hip ratio $(P>.05)$. No significant difference in the body composition among the three groups provided a reasonable basis for explaining the analysis results by controlling variables.

Table 5

Analysis of the difference in Wingate Measurement Indices of Power Bicycle

\begin{tabular}{|c|c|c|c|c|c|c|}
\hline \multicolumn{2}{|c|}{ Indicators } & $\begin{array}{l}\text { Lento }(n=20) \\
M(S D)\end{array}$ & $\begin{array}{l}\text { Moderato }(n=20) \\
M(S D)\end{array}$ & $\begin{array}{l}\text { Allegretto }(n=17) \\
M(S D)\end{array}$ & $F$ & $P$ \\
\hline \multirow[t]{2}{*}{$M P$} & watt & $535.75(170.15)$ & $506.75(164.22)$ & $522.29(143.52)$ & .163 & .850 \\
\hline & Watt/kg & $7.23(1.53)$ & $7.18(1.56)$ & $7.27(1.46)$ & .018 & .982 \\
\hline \multirow[t]{2}{*}{$P P$} & watt & $901.60(304.98)$ & $854.55(394.84)$ & $870.35(267.23)$ & .105 & .900 \\
\hline & watt/kg & $11.96(3.03)$ & $12.69(3.85)$ & $11.85(2.41)$ & .392 & .677 \\
\hline$F I$ & & $22.49(9.70)$ & $24.78(13.36)$ & $21.25(8.43)$ & .512 & .602 \\
\hline$P P I$ & $\sec$ & $4.26(0.28)$ & $4.21(0.24)$ & $4.14(0.40)$ & .669 & .517 \\
\hline$T W$ & watt & $16468.50(4885.61)$ & $15202.50(4926.67)$ & $15668.82(4305.73)$ & .365 & .696 \\
\hline
\end{tabular}

MP: mean power, PP: peak power, MIP: minimum power, PPI: peak power in, Fl: fatigue index, PW: peak power/weight, MW: mean power/weight, TW: total work; $* P<.05$.

Table 5 presents the results of the analysis of differences in the Wingate measurement indices of power bicycle. No significant differences were observed in the relative and absolute values of the average strength and the maximum strength, fatigue index, time of arrival of the maximum force, and real work among the three groups $(P>.05)$. These results confirmed that the difference in high-intensity exercise ability and intensity among the three groups was nonsignificant.

\section{Analysis of HRV time-domain parameters}

\section{Results of time-domain parameter analysis before exercise}


The results of the time-domain variability index analysis before exercise (shown in Table 6) indicated no significant difference in HR and SDNN index among the three groups $(P>.05)$. In addition, the difference in the time-domain index of HRV among the three groups before exercise was found to be non significant.

Table 6

Analysis of time-domain variation index before exercise

\begin{tabular}{|c|c|c|c|c|c|}
\hline Indicators & $\begin{array}{l}\text { lento }(n=20) \\
M(S D)\end{array}$ & $\begin{array}{l}\text { Moderato }(n=20) \\
M(S D)\end{array}$ & $\begin{array}{l}\text { Allegretto }(n=17) \\
M(S D)\end{array}$ & $F$ & $P$ \\
\hline$H R(b p m)$ & $75.20(14.06)$ & $74.90(12.36)$ & $73.29(9.49)$ & .126 & .886 \\
\hline$S D N N(m s)$ & $76.75(41.42)$ & $87.30(36.29)$ & 63.39(33.95) & 1.865 & .165 \\
\hline
\end{tabular}

\section{Results of time-domain variation index analysis in the recovery period}

Table 7

Analysis of time-domain variation index during the recovery period

\begin{tabular}{|c|c|c|c|c|c|c|c|c|}
\hline \multirow[t]{2}{*}{ Indicators } & \multirow{2}{*}{$\begin{array}{l}\text { Heart rate } \\
\text { recovery } \\
\text { time(min) }\end{array}$} & \multirow{2}{*}{$\begin{array}{l}\text { Lento }(n=20) \\
M(S D)\end{array}$} & $\begin{array}{l}\text { Moderato } \\
(n=20)\end{array}$ & $\begin{array}{l}\text { Allegretto } \\
(n=17)\end{array}$ & \multirow[t]{2}{*}{ contrast } & \multirow[t]{2}{*}{$F$} & \multirow[t]{2}{*}{$P$} & \multirow[t]{2}{*}{$\begin{array}{l}\text { Post- } \\
\text { hoc }\end{array}$} \\
\hline & & & $M(S D)$ & $M(S D)$ & & & & \\
\hline \multirow{4}{*}{$\begin{array}{l}\text { HR } \\
(\mathrm{bpm})\end{array}$} & $0-1$ & $168.13(1.50)$ & 166.77(2.49) & 167.15(2.42) & Times & 2020.27 & .000 & \\
\hline & $5-6$ & $106.00(8.80)$ & $99.15(13.39)$ & $98.24(10.61)$ & Groups & $5.548^{*}$ & .006 & $\begin{array}{l}\mathrm{L}> \\
M, L>A\end{array}$ \\
\hline & $10-11$ & $103.85(9.35)$ & $91.60(14.31)$ & $94.06(11.50)$ & \multirow[t]{2}{*}{ Times ${ }^{\star}$ Groups } & \multirow[t]{2}{*}{4.024} & \multirow[t]{2}{*}{.023} & \\
\hline & $15-16$ & $99.05(9.35)$ & $88.40(12.64)$ & $90.59(12.00)$ & & & & \\
\hline \multirow[t]{4}{*}{$S D N N(m s)$} & $0-1$ & $66.20(23.81)$ & $49.05(17.04)$ & $47.24(16.25)$ & Times & 10.709 & .002 & \\
\hline & $5-6$ & $27.03(24.15)$ & $28.40(13.47)$ & $26.00(17.53)$ & Groups & .060 & .942 & NS \\
\hline & $10-11$ & $24.70(26.57)$ & $28.10(15.85)$ & $32.12(25.33)$ & \multirow[t]{2}{*}{ Times*Groups } & \multirow[t]{2}{*}{3.409} & \multirow[t]{2}{*}{.040} & \\
\hline & $15-16$ & $32.65(31.04)$ & $41.90(28.28)$ & $38.00(26.08)$ & & & & \\
\hline
\end{tabular}

The time-domain variation analysis results (presented in Table 7) indicated statistically significant differences in HR among the three groups with different music tempos $(P<.05)$. The result revealed that the HR recovery in the Moderato and Allegretto groups was faster than that in the Lento group. HR recovery was the most prominent in the Moderato group (Figure 1). This finding suggested that listening to music at a moderate speed can most effectively facilitate HR recovery. Although no statistically significant difference was observed in SDNN among the three groups with different tempos $(P>.05)$, the time-domain variation index (shown in Figure 2) revealed that the SDNN values in the Moderato and Allegretto groups were more stable than that in the Lento group. Additionally, the SDNN value in the Moderato group was high and stable. These findings indicated that listening to Moderato piano music can help the ANS maintain a high self-regulation power and facilitate HR recovery during the recovery period.

\section{Analysis of the HRV frequency domain index Results of HRV frequency domain index analysis before exercise}


Table 8

Results of HRV frequency domain index analysis before exercise

\begin{tabular}{|c|c|c|c|c|c|}
\hline Indicators & $\begin{array}{l}\text { Lento }(n=20) \\
M(S D)\end{array}$ & $\begin{array}{l}\text { Moderato }(n=20) \\
M(S D)\end{array}$ & $\begin{array}{l}\text { Allegretto }(n=17) \\
M(S D)\end{array}$ & $F$ & $P$ \\
\hline$L F(m s 2)$ & $7.25(1.05)$ & $6.35(0.80)$ & $6.43(0.66)$ & $6.519 *$ & .003 \\
\hline$H F(m s 2)$ & $6.50(1.10)$ & $6.04(0.90)$ & $5.83(0.84)$ & 2.374 & .103 \\
\hline LFnorm (n.u) & $52.42(3.61)$ & $51.30(3.59)$ & $52.65(3.27)$ & .816 & .448 \\
\hline HFnorm (n.u.) & $47.58(3.61)$ & $48.70(3.59)$ & $47.35(3.27)$ & .816 & .448 \\
\hline$L F / H F$ & $1.13(0.18)$ & $1.07(0.15)$ & $1.12(0.16)$ & .953 & .392 \\
\hline
\end{tabular}

The HRV frequency domain index analysis results before exercise are presented in Table 8. The three groups exhibited no statistically significant difference in HF, LFnorm, HFnorm, and LF/HF frequency domain indices $(P>0.05)$. However, the LF frequency domain index differed significantly among the groups $(P<0.05)$, and the high LF value indicated sympathetic excitement and HR acceleration. Additionally, HR before exercise was found to vary significantly among the groups $(P>.05)$. Thus, the frequency domain index state of HRV before exercise was higher than the standard level.

\section{Results of HRV frequency domain index analysis in the convalescent period}


Table 9

Analysis of HRV frequency domain indices in the convalescent period

\begin{tabular}{|c|c|c|c|c|c|c|c|c|}
\hline Indicators & $\begin{array}{l}\text { Heart rate } \\
\text { recovery } \\
\text { time(min) }\end{array}$ & $\begin{array}{l}\text { lento }(n=20) \\
M(S D)\end{array}$ & $\begin{array}{l}\text { Moderato }(n=20) \\
M(S D)\end{array}$ & $\begin{array}{l}\text { Allegretto }(n=17) \\
M(S D)\end{array}$ & contrast & $F$ & $P$ & $\begin{array}{l}\text { Post- } \\
\text { hoc }\end{array}$ \\
\hline \multirow[t]{4}{*}{$L F(m s 2)$} & $0-1$ & $6.31(1.85)$ & $6.48(1.02)$ & $5.76(1.46)$ & Times & 10.416 & .000 & \\
\hline & $5-6$ & $4.90(1.69)$ & $5.27(1.79)$ & $4.78(1.33)$ & Groups & 1.786 & .177 & $N S$ \\
\hline & $10-11$ & $4.51(1.25)$ & $5.40(1.57)$ & $5.36(1.48)$ & \multirow[t]{2}{*}{ Times ${ }^{\star}$ Groups } & \multirow[t]{2}{*}{.927} & \multirow[t]{2}{*}{.447} & \\
\hline & $15-16$ & $5.49(1.01)$ & $6.12(1.31)$ & $5.79(1.57)$ & & & & \\
\hline \multirow[t]{4}{*}{$H F(m s 2)$} & $0-1$ & $4.84(1.48)$ & $5.45(0.92)$ & $4.74(1.52)$ & Times & 9.486 & .000 & \\
\hline & $5-6$ & $3.85(1.77)$ & $4.40(1.58)$ & $3.84(1.28)$ & Groups & $3.483^{*}$ & .038 & $M>L$ \\
\hline & $10-11$ & $3.67(0.95)$ & $4.63(1.02)$ & $4.03(1.22)$ & \multirow[t]{2}{*}{ Times ${ }^{\star}$ Groups } & \multirow[t]{2}{*}{.893} & \multirow[t]{2}{*}{.501} & \\
\hline & $15-16$ & $4.29(0.94)$ & $4.82(1.30)$ & $5.06(1.14)$ & & & & \\
\hline \multirow[t]{4}{*}{ LFnorm(n.u.) } & $0-1$ & $58.26(3.51)$ & $54.10(3.53)$ & $57.08(4.83)$ & Times & .670 & .571 & \\
\hline & $5-6$ & $55.12(6.50)$ & $54.57(9.26)$ & $56.03(6.62)$ & Groups & .560 & .575 & NS \\
\hline & $10-11$ & $54.82(6.47)$ & $56.68(8.31)$ & $57.49(4.26)$ & \multirow[t]{2}{*}{ Times*Groups } & \multirow[t]{2}{*}{1.393} & \multirow[t]{2}{*}{.220} & \\
\hline & $15-16$ & $55.42(6.32)$ & $56.31(4.08)$ & $56.54(2.86)$ & & & & \\
\hline \multirow[t]{4}{*}{ HFnorm(n.u.) } & $0-1$ & $41.64(3.58)$ & $45.92(3.31)$ & $43.39(4.96)$ & Times & .604 & .613 & \\
\hline & $5-6$ & $44.68(6.35)$ & $45.47(9.24)$ & $43.97(6.62)$ & Groups & .690 & .374 & $N S$ \\
\hline & $10-11$ & $45.19(6.47)$ & $43.15(8.34)$ & $42.51(4.26)$ & \multirow[t]{2}{*}{ Times ${ }^{*}$ Groups } & \multirow[t]{2}{*}{1.647} & \multirow[t]{2}{*}{.143} & \\
\hline & $15-16$ & $44.51(6.31)$ & 43.35(4.03) & $43.46(2.86)$ & & & & \\
\hline \multirow[t]{4}{*}{$L F / H F$} & $0-1$ & $1.37(0.31)$ & $1.20(0.17)$ & $1.31(0.38)$ & Times & .910 & .438 & \\
\hline & $5-6$ & $1.45(0.71)$ & $1.28(0.45)$ & $1.33(0.42)$ & Groups & 1.119 & .334 & $N S$ \\
\hline & $10-11$ & $1.26(0.33)$ & $1.17(0.31)$ & $1.36(0.26)$ & \multirow[t]{2}{*}{ Times ${ }^{\star}$ Groups } & \multirow[t]{2}{*}{1.209} & \multirow[t]{2}{*}{.304} & \\
\hline & $15-16$ & $1.33(0.38)$ & $1.31(0.23)$ & $1.13(0.13)$ & & & & \\
\hline
\end{tabular}

The HRV frequency domain index analysis results are presented in Table 9. The LF frequency domain indices were not found to differ significantly among the three groups exposed to varying rhythms during the recovery period $(P>.05)$. With higher values of LF and LFnorm, the athlete's sympathetic nerves excited more and the heartbeat was faster. The results indicate that the piano music tempo cannot have a significant effect on LF.

A statistically significant difference was noted in the HF frequency domain index among different repertoire groups during the recovery period $(P<0.05)$. However, no significant difference was observed in the HFnorm frequency domain index among the groups $(P>0.05)$.

The HF curve chart of frequency domain variation index is presented in Figure 3. The HF value in the Moderato group was found to be higher than those in the Allegretto and Lento groups. Therefore, listening to music of Moderato speed can increase the excitability of the parasympathetic nerve, decrease the HR, and calm the HR quickly after high-intensity exercise compared with Allegretto and Lento tempos.

The line chart of the frequency domain variation index LF/HF is depicted in Figure 4. The LF/HF ratio in the Moderato group was found to be smaller than those in the Allegretto and Lento groups, although no statistically significant difference was observed in 
the frequency domain index of LF/HF $(P>0.05)$. Moderato speed tunes contributed to the recovery of the balance between sympathetic and parasympathetic nerves after high-intensity exercise.

\section{Discussion}

In the present study, we found that under the same exercise intensity, the body composition, high-intensity exercise ability, timedomain variation indices (HR and SDNN) before exercise, frequency domain indices (HF, LFnorm, HFnorm, LF/HF, and HRV index of HRV) before exercise do not differ significantly across different groups exposed to varying rhythms $(P>.05)$. Studies have confirmed that the more excited the parasympathetic nerve (vagus nerve) is, the more acetylcholine it releases and the more it acts on the $\mathrm{M}$ receptor on the myocardial surface, which effectively inhibits the myocardial excitement. In addition, the vagus nerve can effectively inhibit the function of the sinoatrial node and cardiac electrical conduction, as well as reduce the HR ${ }^{14}$. The external factors imposed during the recovery period were piano music of different tempos, which might be restricted by other external factors. However, we maintained a constant temperature and humidity in the laboratory during experiments, and the time of the experiment was set between 14:00 and 15:00 hours of the day. The three people were analyzed every day to reduce the impact of external factors on the research results. Therefore, no significant differences in physiology and motor ability among the groups were evident. By analyzing time-domain variations in the recovery period, significant differences were observed in the HR among the groups exposed to different music tempos $(P<0.05)$. The analysis of the time-domain variation index, HR, revealed that the HR recovery in the Moderato and Allegretto groups was faster than that in the Lento group, and the recovery in the Moderato group was the most prominent. The finding suggests that listening to the repertoire of Moderato speed is the most effective means for enhancing HR recovery in the recovery period. However, no significant difference was observed in SDNN among the groups $(P>.05)$. The index of time-domain variation revealed that the values of SDNN in the Moderato and Allegretto groups were more stable than that in the Lento group, and the SDNN value in the Moderato group was the highest and most stable. Thus, compared with the other two groups of piano music, listening to piano music with Moderato speed can help the ANS in maintaining a high self-regulation force more efficiently and accelerate the recovery of HR during the recovery period ${ }^{15-17}$. The analysis of the HRV frequency domain index in the recovery period indicated that although the high values of LF and LFnorm reflect excited state of the sympathetic nerve, norepinephrine release at the end of the sympathetic nerve, enhancement of myocardial activity, and acceleration of HR caused by $\beta$-adrenoceptor, the LF frequency domain index did not differ significantly among the piano music groups exposed to different tempos during the recovery period $(P>0.05)$. The tempo of the music did not have a significant effect on LF. Although the HFnorm frequency domain index did not differ significantly among the groups $(P>$ 0.05), a significant difference was observed in the HF frequency domain index among different velocity curve groups during the recovery period $(P<0.05)$, HF reflected the activity of the parasympathetic nerve. The more excited the parasympathetic nerve (vagus nerve) is, the more acetylcholine it releases at the end of the parasympathetic nerve, which acts on the muscarinic receptor on the myocardium surface and inhibits the excitement of the myocardium ${ }^{18-21}$.In addition, the vagus nerve inhibits the function of the sinoatrial node and cardiac electrical conduction and reduces the HR. The value of HF in the Moderato group was higher than those in the Allegretto and Lento groups; therefore, listening to Moderato-speed music can increase parasympathetic excitability, decrease HR, and calm HR quickly after high-intensity exercise compared with Allegretto and Lento tempos. The lowfrequency and high-frequency power ratio (LF/HF) reflects the balance between the sympathetic and parasympathetic nerves ${ }^{22-}$

24 . Although the LF/HF frequency domain index did not differ significantly among the groups $(P>0.05)$, the line chart of the average change of frequency domain variation index LF/HF in the recovery period indicated that the LF/HF ratio in the Moderato group was smaller than those in the Allegretto and Lento groups.

\section{Conclusions}

The findings of this study indicate that after excluding external factors, the repertoire of Moderato speed contributes to restore the balance between sympathetic and parasympathetic nerves after high-intensity exercise. Thus, moderate-tempo piano music can be used to accelerate fatigue recovery after high-intensity exercise in any individual performing high-intensity exercise.

\section{Declarations}


None.

\section{Authors' contributions}

Study design: Xue Biyun, Wang Jiameng

Data collection: Xue Biyun, Wang Jiameng

Data analysis: Wang Jiameng

Study supervision: Xue Biyun, Wang Jiameng

Manuscript writing: Critical revisions for important intellectual content: Xue Biyun, Wang Jiameng

Wang Jiameng is the corresponding authors, For the correspondence and telephone number of the paper, please contact Professor Wang at the following addresses: Department of Physical Education, Hainan Medical University, Haikou,Hainan 571199, China;E-mail address: hy0215037@hainmc.edu.cn

\section{Funding}

This study was funded by Scientific Research Project of Higher Education Institutions of Hainan Province(Hnky2021-41).

\section{Conflict of interest}

No conflict of interest has been declared by the authors

\section{References}

1. Pengyi, D. \& Changlin, H.research progress of exercise-induced fatigue. Medical Journal of the people's Liberation Army.41,955-964 (2016).

2. Shuxun, D., Hong Taitian, H. \& Zhifa, C.Exercise physiology. Beijing: higher Education Press.346; 10.1249/00005768199503000-00027(2001).

3. Rong, L., Wenkai, J., Jing, L. \& Jinghua J. A preliminary study on the effects of intermittent hypoxia training, fatigue and recovery on $\mathrm{H}$ reflex parameters. Sports and Science.31,88-91+ 41 (2010).

4. Tiantian, J., Yoshiko, O. \& Misa, M.Music Attenuated a Decrease in Parasympathetic Nervous System Activity after Exercise. Plos One,11,e0148648(2016).

5. Imai, K., Sato, H., Hori, M., Kusuoka, H., Ozaki, H. \& Yokoyama, H. Vagally mediated heart rate recoveryafter exercise is accelerated In athletes but blunted in patients with chronic heart failure. Journal of the American College of Cardiology.24,1529-1535 (1994).

6. Desempenho, D.M.Autonomic contribution to heart rate recovery from exercise in human.J Appl Physiol Respir Environ Exerc Physiol.53, 1572-1575 (1892).

7. Cole, C.R., Blackstone, E.H. \& Pashkow, F.J.Heart-Rate Recovery Immediately after Exercise as a Predictor of Mortality. N Engl J Med.341,1351-1357 (1999).

8. Goldberger, J.J., Challapalli, S. \& Tung, R.Relationship of Heart Rate Variability to Parasympathetic Effect. Circulation.103,1977-1983 (2001).

9. Stefan, K. \& Jäncke, L.Music and the heart. European Heart Journal.36,3043-3049 (2015).

10. Iwanaga, M., Kobayashi, A. \& Kawasaki, C.Heart rate variability with repetitive exposure to music. Biological Psychology.70,61-66 (2005).

11. Orini, M., Raquel, B. \& Enk, R.A method for continuously assessing the autonomic response to music-induced emotions through HRV analysis. Med Biol Eng Comput.48, 423-433 (2010). 
12. Yamashita, S., Iwai, K. \& Akimoto, T. Effects of music during exercise on RPE, heart rate and the autonomic nervous system.Journal of Sports Medicine and Physical Fitness.46,425-430 (2006).

13. Urakawa, K. \& Yokoyama, K.Music Can Enhance Exercise-Induced Sympathetic Dominancy Assessed by Heart Rate Variability. The Tohoku Journal of Experimental Medicine.206,213-218 (2005).

14. Macor, F., Fagard, R. \& Amery, A.Power spectral analysis of RR interval and blood pressure short-term variability at rest and during dynamic exercise: comparison between cyclists and controls. Int J Sports Med.17,175-181 (1996).

15. Kuo, T.B., Lin, T., Yang, C.C., Li, C.L., Chen, C.F. \& Chou, P.Effect of aging on gender differences in neural control of heart rate. Am J Physiol.277,H2233-2239 (1999).

16. Houle, M.S. \& Billman, G.E.Low-frequency component of the heart rate variability spectrum: a poor marker of sympathetic activity. Am J Physiol.276,215-223 (1999).

17. Sumi, K., Suzuki, S., Matsubara, M., Ando, Y. \& Kobayashi, F. Heart rate variability during high-intensity fieldexercise in female distance runners. Scandinavian journal of medicine and science in sports.16,314-320 (2006).

18. Ekblom, B., Kilbom, A. \& Soltysiak, J.Physical training, bradycardia, and autonomic nervous system. Scandinavian Journal of Clinical \& Laboratory Investigation.32,.251-256 (1973).

19. Goldsmith, R.L., Bigger, J.T. \& Steinman, R.C.Comparison of 24-hour parasympathetic activity in endurance-trained and untrained young men.Journal of the American College of Cardiology.32,552-558(1992).

20. Puig, J., Freitas, J. \& Carvalho, N.Spectral analysis of heart rate variability in athletes. Journal of Sports Medicine and Physical Fitness.33,44-48 (1993).

21. Shin, K., Minamitani, H. \& Onishi, S.Autonomic differences between athletes and nonathletes: spectral analysis approach. Medicine and Science in Sports and Exercise.29,1482-1490 (1997).

22. Rinci, T., Finderle, Z. \& Parco S.Gender-related cardiac autonomic modulation in young lite athletes. Acta Physiologica.197135 (2002).

23. Tulppo, M.P., Mäkikallio,T.H. \& Takala, T.E.Quantitative beat-to-beat analysis of heart rate dynamics during exercise. American Journal of Physiology.271,H244-252 (1996).

24. Princi, T., Fabbro, I.D., Peterec, M., Fonda, L.C. \& Accardo, A. Non-linear Assessment of Heart Rate Variability in Ovolactovegetarians, Vegans and Omnivores during Oral Glucose Tolerance Test.14th Nordic-Baltic Conference on Biomedical Engineering and Medical Physics.14,315-318 (2008).

\section{Figures}

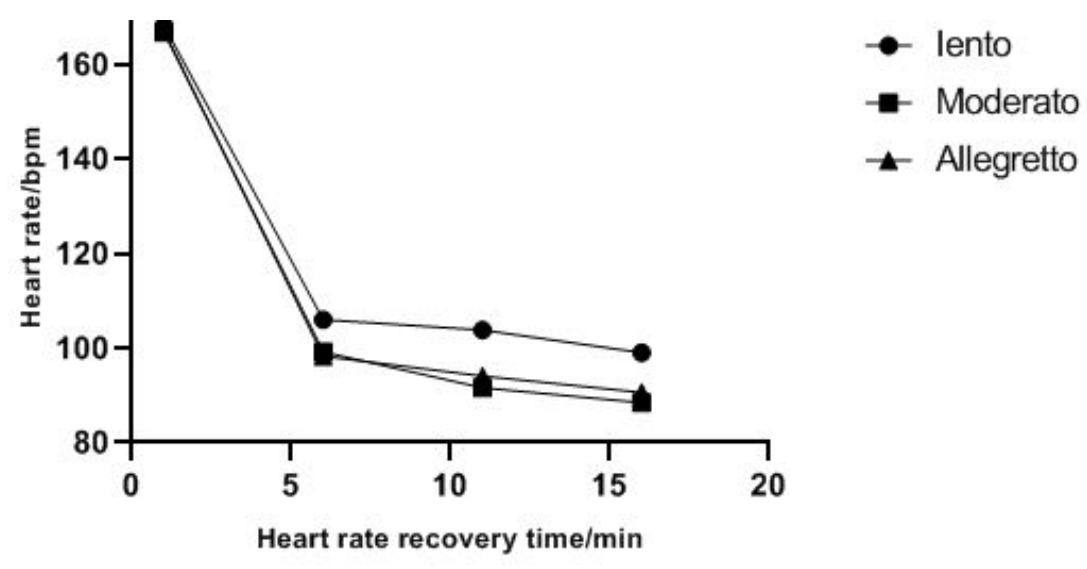

\section{Figure 1}

The time-domain variation index HR in the recovery period 


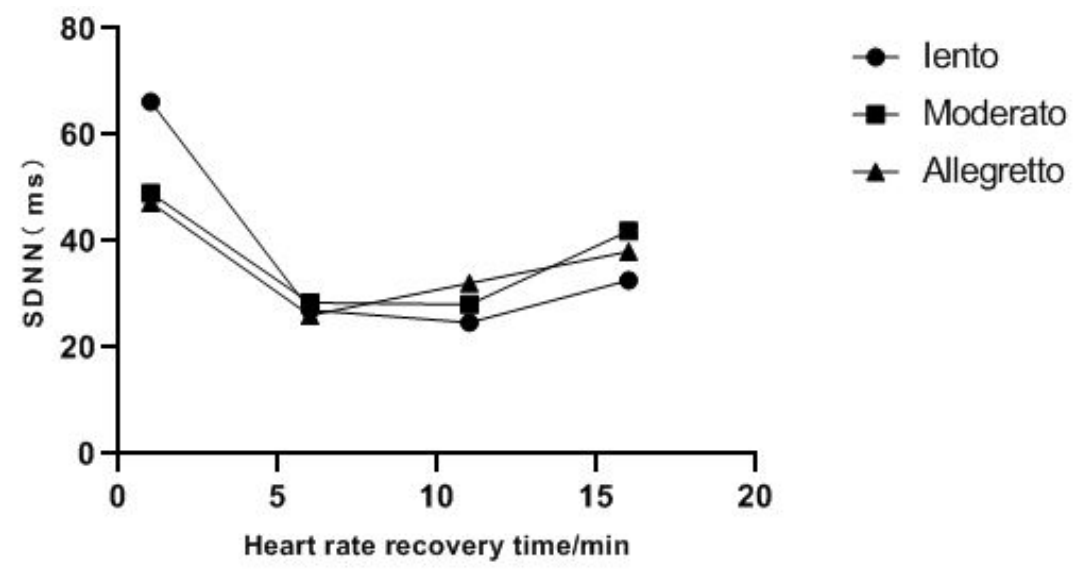

Figure 2

The time-domain variation index SDNN in the recovery period

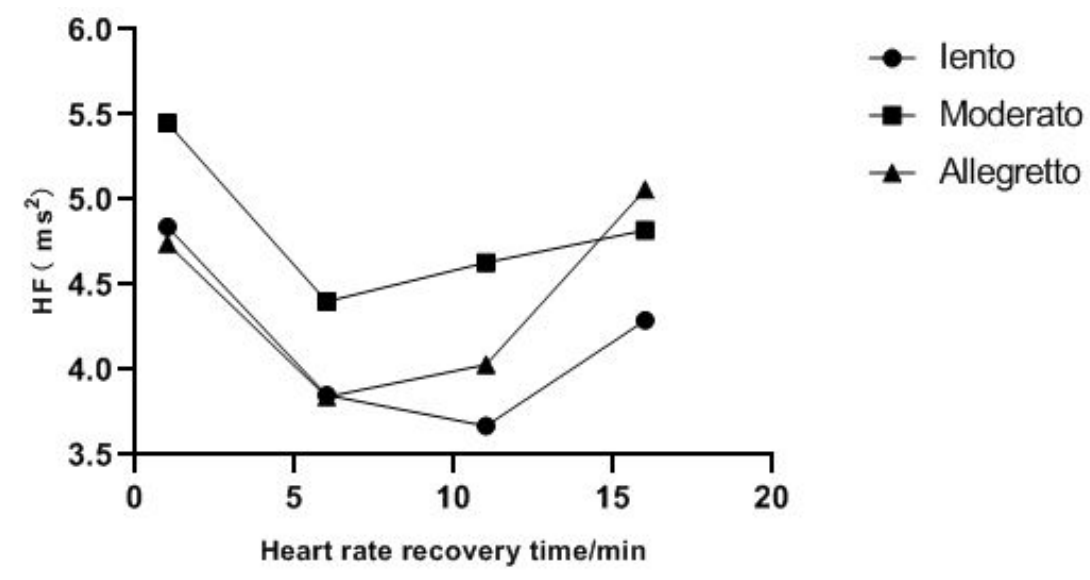

Figure 3

The time-domain variation index HF in the recovery period

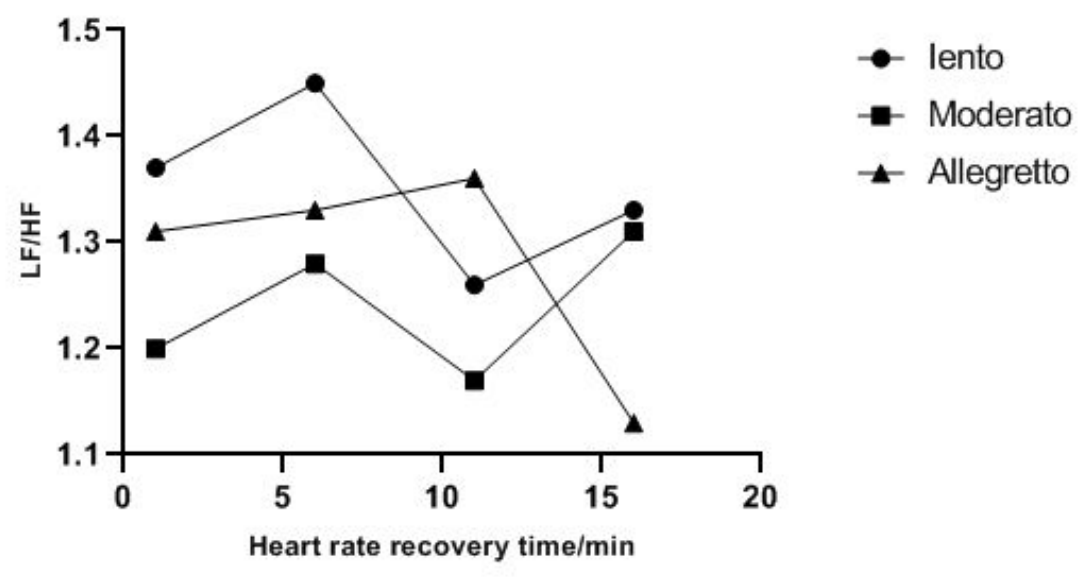

Figure 4

The time-domain variation index LF/ HF in the recovery period 